\title{
Predictive functional control based on fuzzy model: magnetic suspension system case study
}

\author{
Marko Lepetič $^{\mathrm{a}, *}$, Igor Škrjanc ${ }^{\mathrm{a}}$, Hector G. Chiacchiarini ${ }^{\mathrm{b}}$, Drago Matko ${ }^{\mathrm{a}}$ \\ ${ }^{a}$ Laboratory of Process Automation and Information, Faculty of Electrical Engineering, University of Ljubljana, Tržaška 25, \\ SI-1000 Ljubljana, Slovenia \\ ${ }^{\mathrm{b}}$ Department of Electrical Engineering, National University of the South, Avda. Alem 1253, (8000) Bahia Blanca, Argentina
}

Received 11 February 2003; accepted 11 June 2003

\begin{abstract}
Fuzzy model based predictive functional controller (FPFC) is applied to the magnetic suspension system - a pilot plant for magnetic bearing. High quality control requirements are short settle time with a-periodical step response and zero steady-state error. Open loop unstable process was stabilised with linear lead compensator. The FPFC was used as a cascade controller. Due to some model uncertainties, the Takagi-Sugeno fuzzy model of stabilised system was obtained using fuzzy identification. Comparing to PID, it improved quality and robustness performance. With its computational efficiency, it proved to be ideal solution for high sampling frequency systems.
\end{abstract}

(C) 2003 Elsevier Ltd. All rights reserved.

Keywords: Fuzzy identification; Predictive control; Real-time control

\section{Introduction}

Predictive control is the name for several different control methods such as: generalized predictive control (GPC) (Clarke et al., 1987), dynamics matrix control (DMC) (Cutler and Ramaker, 1980) and predictive functional control (PFC) (Richalet et al., 1978). The control law is based on the prediction, obtained with the model of the controlled process. Control action is calculated in the way to minimize the difference between the predicted process output and the reference signal over a certain time horizon. Predictive controllers generally exhibit remarkable robustness with the respect to the model mismatch and unmodelled dynamics (Camacho and Bordons, 1995). Very good results were also achieved in combination with time delay processes (Camacho and Bordons, 1995). When based on fuzzy model, predictive controllers proved to be very convenient for strongly nonlinear processes (Škrjanc and Matko, 2000; Škrjanc and Matko, 2001).

\footnotetext{
*Corresponding author. Tel.: + 386-1-476-87-02; fax: + 386-1-42646-31.

E-mail address: marko.lepetic@fe.uni-lj.si (M. Lepetič).
}

The researches that have been made and are presented in this paper are related to the idea of magnetic bearing. Very high rotational frequency can only be reached if bearing causes very low friction. The best way to solve this problem is to avoid physical contact. Using the magnetic force there are two possibilities to hold an object at the certain position. If the magnetic force repulses floating object we have to deal with stable system. Such realization causes construction problem because it requires one electromagnet in the rotor that is turning. If the rotor electromagnet is replaced by ferromagnetic coil, then the force could only be attracting and the system become unstable. Besides stability, requirements are short settle time with a-periodical step response and zero steady-state error. The a-periodical step response is especially important to avoid the transfer of oscillations caused by rotation to the position of the rotor. Requirements were fulfilled with the cascade control using linear lead compensator in inner loop and predictive controller in outer loop. Inner loop assures stability, but cannot eliminate steady-state error and also causes overshoot on a step response. Outer loop PFC based on fuzzy model improves performance of the system. It solves both mentioned problems and makes reference trajectory tracking almost perfect. 
The paper is organized follows: The magnetic suspension system is presented in Section 2. Section 3 describes the concept of fuzzy identification. PFC principles and design are given in Section 4 and the real-time implementation of control algorithm together with the comparative analysis with the PID controller on the magnetic suspension system is presented in Section 5.

\section{Magnetic suspension system}

Magnetic suspension system consists of an electromagnet, a coil and a distance sensor. Its basic principle is shown in Fig. 1, where $u_{R L}$ and $i$ stand for voltage and current of the electromagnet respectively, $R$ and $L$ are resistance and inductance of the electromagnet, $c$ is unknown parameter, $m$ is the mass of the coil and $l$ is the distance between the electromagnet and the coil. The goal is to control the distance $l$ by the control variable $u_{\mathrm{RL}}$.

Using the second Newton law we can write:

$m g-F_{\mathrm{m}}=m \frac{\mathrm{d}^{2} l}{\mathrm{~d} t^{2}}$.

The magnetic force depends on the current $i$, the distance $l$ and the parameter $c$ :

$F_{\mathrm{m}}=c \frac{i^{2}}{l^{2}}$.

The electrical part of the system is modelled with the following equation:

$u_{R L}(t)=L \frac{\mathrm{d} i}{\mathrm{~d} t}+R i$

The sensor and the actuator can be modelled with static functions where Eq. (4) is the model of the sensor and Eq. (5) is model of the actuator:

$$
\begin{aligned}
& y_{\mathrm{p}}=K_{\text {sens }} l+U_{\text {sens }}, \quad K_{\text {sens }}=-4 \mathrm{~V} / \mathrm{mm}, \\
& U_{\text {sens }}=10 \mathrm{~V}, \\
& u= K_{\text {act }} u_{R L}+U_{\text {act }}, \quad K_{\text {act }}=2, \quad U_{\text {act }}=-10 \mathrm{~V} .
\end{aligned}
$$

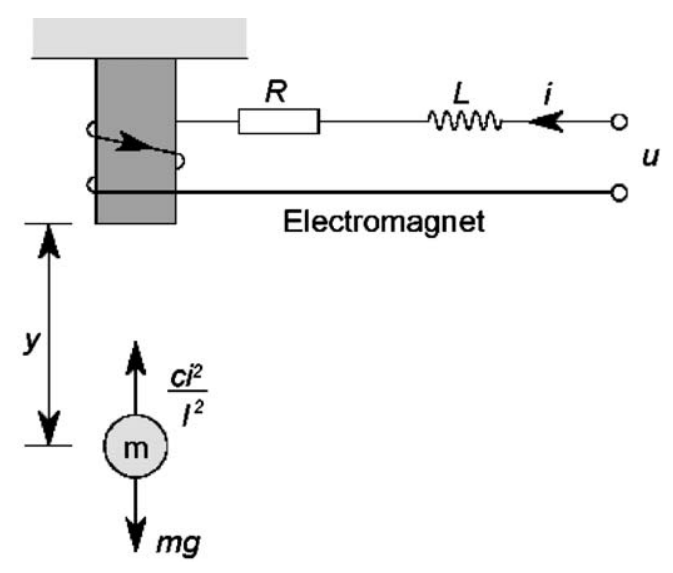

Fig. 1. Basic principle of magnetic suspension.
By combining Eqs. (1)-(3) the nonlinear unstable differential equation is obtained

$$
\begin{aligned}
& \frac{L m}{c}\left(g-\frac{\mathrm{d}^{2} l}{\mathrm{~d} t^{2}}\right) \frac{\mathrm{d} l}{\mathrm{~d} t}-\frac{L m}{2 c} l \frac{\mathrm{d}^{3} l}{\mathrm{~d} t^{3}} \\
& \quad+\frac{R m}{c} l\left(g-\frac{\mathrm{d}^{2} l}{\mathrm{~d} t^{2}}\right)-u_{R L} \sqrt{\frac{m}{c}\left(g-\frac{\mathrm{d}^{2} l}{\mathrm{~d} t^{2}}\right)}=0 .
\end{aligned}
$$

It can be stabilized with the linear lead compensator with transfer function:

$$
G_{\mathrm{cc}}(s)=4.5\left(\frac{s+40}{s+400}\right)
$$

which corresponds at $2 \mathrm{~ms}$ sampling time to discrete transfer function:

$G_{\mathrm{cd}}\left(z^{-1}\right)=4.5\left(\frac{1-0.9449 z^{-1}}{1-0.4493 z^{-1}}\right)$.

Also the feed-forward compensation of gravity of $2 \mathrm{~V}$ was applied.

Robustness of the lead compensator assures the stability in the whole operating range. It is well known that the steady-state error cannot be eliminated by just using the lead compensator, therefore an additional fuzzy PFC was added in the cascade. To design the outer loop fuzzy predictive controller, the fuzzy model of the inner loop had to be obtained. Due to the unknown values of $R, L, c$ and nonlinear nature of the observed process, a fuzzy identification was used to obtain the process model.

\section{Fuzzy identification}

The prediction is made on a global linear model obtained from TS fuzzy model, (Sugeno and Tanaka, 1991; Takagi and Sugeno, 1985) so let us take a quick overview of it. The $j$ th rule of the TS model can be written as follows:

$$
\begin{aligned}
& \mathbf{R}^{j}: \text { if } x_{1} \text { is } A_{1}^{j} \text { and } \ldots \text { and } x_{N} \text { is } A_{N}^{j} \\
& \text { then } y=f^{j}\left(x_{1}, \ldots, x_{N}\right)
\end{aligned}
$$

where $x_{i}$ are inputs, $A_{i}^{j}$ are subsets of the input space, $y$ is the output and $f^{j}$ is a function, generally nonlinear.

In case of the magnetic suspension, the model is determined in the form of the TS fuzzy model. A minor modification has been made in the sense that the antecedent variable is not a part of the regressor. For the third order model, $i$ th rule can be written as

$$
\begin{aligned}
& \mathbf{R}^{i}: \text { if av is } A^{i} \text { then } \\
& \begin{aligned}
y_{\mathrm{p}}(k+1)= & a_{1 i} y_{\mathrm{p}}(k)+a_{2 i} y_{\mathrm{p}}(k-1)+a_{3 i} y_{\mathrm{p}}(k-2) \\
& +b_{i} u(k-D)+r_{i},
\end{aligned}
\end{aligned}
$$


where $y_{\mathrm{p}}(k+1)$ is the output and $y_{\mathrm{p}}(k), y_{\mathrm{p}}(k-1)$, $y_{\mathrm{p}}(k-2), u(k-D)$ are the inputs of the fuzzy model. $D$ stands for the dead time expressed by the number of samples, $A^{i}$ are antecedent fuzzy sets and av is the antecedent variable.

Using fuzzy mean defuzzification method, the output is expressed by the following equation:

$$
\begin{aligned}
y_{\mathrm{p}}(k+1)= & \sum_{i=1}^{K} \beta_{i}(k)\left(a_{1 i} y(k)+a_{2 i} y(k-1)\right. \\
& \left.+a_{3 i} y(k-2)+b_{i} u(k-D)+r_{i}\right),
\end{aligned}
$$

where $K$ stands for the number of rules and $\beta_{i}(k)$ is the normalized degree of fulfilment of $i$ th rule at $k$ th step.

The vector of parameters of $i$ th rule $\boldsymbol{\theta}_{i}$ is obtained by using the least-squares method.

$\boldsymbol{\theta}_{i}=\left(\boldsymbol{\Psi}_{i}^{\mathrm{T}} \boldsymbol{\Psi}_{i}\right)^{-1} \boldsymbol{\Psi}_{i}^{\mathrm{T}} \mathbf{Y}_{p}^{i}$,

where the elements of $\boldsymbol{\theta}_{i}$ are $a_{1 i}, a_{2 i}, a_{3 i}, b_{i}$ and $r_{i}$.

$\boldsymbol{\theta}_{i}^{\mathrm{T}}=\left[\begin{array}{lllll}a_{1 i} & a_{2 i} & a_{3 i} & b_{i} & r_{i}\end{array}\right]$.

Vectors $\boldsymbol{\theta}_{i}$ can be joined to a matrix of parameters

$\boldsymbol{\Theta}=\left[\begin{array}{llll}\boldsymbol{\theta}_{1} & \boldsymbol{\theta}_{2} & \cdots & \boldsymbol{\theta}_{K}\end{array}\right]$,

where $i$ th column represents the parameter vector of $i$ th rule. The fuzzy model of the Eq. (11) can be written in the following form also called global linear model

$$
\begin{aligned}
y_{\mathrm{p}}(k+1)= & \tilde{a}_{1}(k) y_{\mathrm{p}}(k)+\tilde{a}_{2}(k) y_{\mathrm{p}}(k-1) \\
& +\tilde{a}_{3}(k) y_{\mathrm{p}}(k-2) \\
& +\tilde{b}(k) u(k-D)+\tilde{r}(k),
\end{aligned}
$$

where the parameters are

$$
\begin{aligned}
& \tilde{a}_{1}(k)=\sum_{i=1}^{K} \beta_{i}(k) \boldsymbol{\Theta}_{1 i}, \\
& \tilde{a}_{2}(k)=\sum_{i=1}^{K} \beta_{i}(k) \boldsymbol{\Theta}_{2 i}, \\
& \tilde{a}_{3}(k)=\sum_{i=1}^{K} \beta_{i}(k) \Theta_{3 i}, \\
& \tilde{b}(k)=\sum_{i=1}^{K} \beta_{i}(k) \boldsymbol{\Theta}_{4 i}, \\
& \tilde{r}(k)=\sum_{i=1}^{K} \beta_{i}(k) \boldsymbol{\Theta}_{5 i} .
\end{aligned}
$$

\section{Fuzzy PFC}

The basic idea of model-based predictive control is to predict the future behaviour of the process over a certain horizon using the dynamic model and obtaining the control actions to minimize a certain criterion, generally

$$
\begin{aligned}
J(u, k)= & \sum_{j=N_{1}}^{N_{2}}\left(y_{\mathrm{m}}(k+j)-y_{\mathrm{r}}(k+j)\right)^{2} \\
& +\lambda \sum_{j=1}^{N_{u}} u^{2}(k+j) .
\end{aligned}
$$

Signals $y_{\mathrm{m}}(k+j), y_{\mathrm{r}}(k+j), u(k+j)$ are $j$-step ahead predictions of the process output, the reference trajectory and the control signal, respectively. Parameter $\lambda$ is the weight of the control signal energy. $N_{1}, N_{2}$ and $N_{u}$ are minimum, maximum and control horizon respectively.

PFC is one of MBPC method. The time-consuming optimisation is included implicitly in the control law, so PFC is a very appropriate method for short sampling time processes. In combination with fuzzy model is called Fuzzy PFC (FPFC).

The PFC is designed in the time domain. For the purpose of $H$ step-ahead prediction, the model from the Eq. (15) should be transformed into a more compact form, for example in the state space domain

$\mathbf{x}_{\mathrm{m}}(k+1)=\tilde{\mathbf{A}}_{\mathrm{m}} \mathbf{x}_{\mathrm{m}}(k)+\tilde{\mathbf{B}}_{\mathrm{m}} u(k)+\tilde{\mathbf{R}}_{\mathrm{m}}$,

$y_{\mathrm{m}}(k)=\tilde{\mathbf{C}}_{\mathrm{m}} \mathbf{x}_{\mathrm{m}}(k)$.

If the state vector $\mathbf{x}_{\mathrm{m}}(k)$ is

$\mathbf{x}_{\mathrm{m}}(k)=\left[\begin{array}{c}y_{\mathrm{m}}(k) \\ y_{\mathrm{m}}(k-1) \\ y_{\mathrm{m}}(k-2)\end{array}\right]$,

then matrices $\tilde{\mathbf{A}}_{\mathrm{m}}, \tilde{\mathbf{B}}_{\mathrm{m}}, \tilde{\mathbf{R}}_{\mathrm{m}}$ and $\tilde{\mathbf{C}}_{\mathrm{m}}$, become

$$
\begin{aligned}
& \tilde{\mathbf{A}}_{\mathrm{m}}=\left[\begin{array}{ccc}
\tilde{a}_{1} & \tilde{a}_{2} & \tilde{a}_{3} \\
1 & 0 & 0 \\
0 & 1 & 0
\end{array}\right], \\
& \tilde{\mathbf{B}}_{\mathrm{m}}=\left[\begin{array}{c}
\tilde{b} \\
0 \\
0
\end{array}\right],
\end{aligned}
$$

$\tilde{\mathbf{R}}_{\mathrm{m}}=\left[\begin{array}{c}\tilde{r} \\ 0 \\ 0\end{array}\right]$,

$\tilde{\mathbf{C}}_{\mathrm{m}}=\left[\begin{array}{lll}1 & 0 & 0\end{array}\right]$.

Presuming $u(k)=u(k+1)=\cdots=u(k+H-1)$, the $H$ step-ahead prediction can be written

$$
\begin{aligned}
y_{\mathrm{m}}(k+H)= & \tilde{\mathbf{C}}_{\mathrm{m}}\left(\tilde{\mathbf{A}}_{\mathrm{m}}^{H} \mathbf{x}_{\mathrm{m}}(k)+\left(\tilde{\mathbf{A}}_{\mathrm{m}}^{H-1}+\cdots+\tilde{\mathbf{A}}_{\mathrm{m}}+\mathbf{I}\right)\right. \\
& \left.\times\left(\tilde{\mathbf{B}}_{\mathrm{m}} u(k)+\tilde{\mathbf{R}}_{\mathrm{m}}\right)\right) .
\end{aligned}
$$


The sum of powered $\tilde{\mathbf{A}}_{\mathrm{m}}$ matrices can be simplified as $\tilde{\mathbf{A}}_{\mathrm{m}}^{H-1}+\cdots+\tilde{\mathbf{A}}_{\mathrm{m}}+\mathbf{I}=\left(\tilde{\mathbf{A}}_{\mathrm{m}}^{H}-\mathbf{I}\right)\left(\tilde{\mathbf{A}}_{\mathrm{m}}-\mathbf{I}\right)^{-1}$.

The closed-loop response should be similar to the reference trajectory, which is the output of the reference model:

$\mathbf{x}_{\mathrm{r}}(k+1)=\mathbf{A}_{\mathrm{r}} \mathbf{x}_{\mathrm{r}}(k)+\mathbf{B}_{\mathrm{r}} w(k)$,

$y_{\mathrm{r}}(k)=\mathbf{C}_{\mathrm{r}} \mathbf{x}_{\mathrm{r}}(k)$.

Matrices $\mathbf{A}_{\mathrm{r}}, \mathbf{B}_{\mathrm{r}}$ and $\mathbf{C}_{\mathrm{r}}$ have to be chosen to fulfil the equation:

$\mathbf{C}_{\mathrm{r}}\left(\mathbf{I}-\mathbf{A}_{\mathrm{r}}\right)^{-1} \mathbf{B}_{\mathrm{r}}=1$.

In the same manner, as with the process model, the $H$ step-ahead prediction of the reference model can be written as

$$
\begin{aligned}
y_{\mathrm{r}}(k+H)= & \mathbf{C}_{\mathrm{r}}\left(\mathbf{A}_{\mathrm{r}}^{H} \mathbf{x}_{\mathrm{r}}(k)\right. \\
& \left.+\left(\mathbf{A}_{\mathrm{r}}^{H}-\mathbf{I}\right)\left(\mathbf{A}_{\mathrm{r}}-\mathbf{I}\right)^{-1} \mathbf{B}_{\mathrm{r}} w(k)\right) .
\end{aligned}
$$

The main goal of FPFC is to equalize the process objective increment $\Delta_{\mathrm{p}}$ and the model objective increment $\Delta_{\mathrm{m}}$ at a certain horizon $H$. The process objective increment is the difference between $H$-step-ahead predicted reference trajectory and the present process output

$\Delta_{\mathrm{p}}=y_{\mathrm{r}}(k+H)-y_{\mathrm{p}}(k)$,

$$
\begin{aligned}
\Delta_{\mathrm{p}}= & \mathbf{C}_{\mathrm{r}}\left(\mathbf{A}_{\mathrm{r}}^{H} \mathbf{x}_{\mathrm{r}}(k)\right. \\
& \left.+\left(\mathbf{A}^{H}-\mathbf{I}\right)\left(\mathbf{A}_{\mathrm{r}}-\mathbf{I}\right)^{-1} \mathbf{B}_{\mathrm{r}} w(k)\right)-y_{\mathrm{p}}(k) .
\end{aligned}
$$

The model output increment is defined as

$$
\begin{aligned}
\Delta_{\mathrm{m}}= & y_{\mathrm{m}}(k+H)-y_{\mathrm{m}}(k), \\
\Delta_{\mathrm{m}}= & \tilde{\mathbf{C}}_{\mathrm{m}}\left(\tilde{\mathbf{A}}_{\mathrm{m}}^{H} \mathbf{x}_{\mathrm{m}}(k)\right. \\
& \left.+\left(\tilde{\mathbf{A}}_{\mathrm{m}}^{H}-\mathbf{I}\right)\left(\tilde{\mathbf{A}}_{\mathrm{m}}-\mathbf{I}\right)^{-1}\left(\tilde{\mathbf{B}}_{\mathrm{m}} u(k)+\tilde{\mathbf{R}}_{\mathrm{m}}\right)\right) \\
& -y_{\mathrm{m}}(k) .
\end{aligned}
$$

As mentioned above the control action is obtained by equalizing

$\Delta_{\mathrm{p}}=\Delta_{\mathrm{m}}$.

By deriving the control variable $u(k)$, the control law can be explicitly expressed as

$$
\begin{aligned}
u(k)= & \frac{\mathbf{C}_{\mathrm{r}} \mathbf{A}_{\mathrm{r}}^{H} \mathbf{x}_{\mathrm{r}}(k)+\mathbf{C}_{\mathrm{r}}\left(\mathbf{A}_{\mathrm{r}}^{H}-\mathbf{I}\right)\left(\mathbf{A}_{\mathrm{r}}-\mathbf{I}\right)^{-1} \mathbf{B}_{\mathrm{r}} w(k)-y_{\mathrm{p}}(k)}{\tilde{\mathbf{C}}_{\mathrm{m}}\left(\tilde{\mathbf{A}}_{\mathrm{m}}^{H}-\mathbf{I}\right)\left(\tilde{\mathbf{A}}_{\mathrm{m}}-\mathbf{I}\right)^{-1} \tilde{\mathbf{B}}_{\mathrm{m}}} \\
& -\frac{\tilde{\mathbf{C}}_{\mathrm{m}} \tilde{\mathbf{A}}_{\mathrm{m}}^{H} \mathbf{x}_{\mathrm{m}}(k)-\tilde{\mathbf{C}}_{\mathrm{m}}\left(\tilde{\mathbf{A}}_{\mathrm{m}}^{H}-\mathbf{I}\right)\left(\tilde{\mathbf{A}}_{\mathrm{m}}-\mathbf{I}\right)^{-1} \tilde{\mathbf{R}}_{\mathrm{m}}+y_{\mathrm{m}}(k)}{\tilde{\mathbf{C}}_{\mathrm{m}}\left(\tilde{\mathbf{A}}_{\mathrm{m}}^{H}-\mathbf{I}\right)\left(\tilde{\mathbf{A}}_{\mathrm{m}}-\mathbf{I}\right)^{-1} \tilde{\mathbf{B}}_{\mathrm{m}}} .
\end{aligned}
$$

\section{Real-time experiment}

The theory of FPFC has been applied to the magnetic suspension previously stabilized with the lead compensator. The electrical part of the system from Eq. (3) has very fast dynamics so the poles from Eq. (1) are dominant. We can presume that the magnetic suspension is originally a second-order system. The compensator adds another pole into the closed-loop system, which results in the closed-loop structure of the third order.

According to the different dynamics dependent on the trend of the distance, the antecedent variable av was chosen to be the filtered derivative of the process output $\mathrm{av}=y_{\mathrm{p}}(k)-y_{\mathrm{p}}(k-10)$. The best identification result was achieved when using the following structure of the TS model.

TS model has antecedent fuzzy sets $A_{1}$ and $A_{2}$. Membership functions of antecedent fuzzy sets are shown in Fig. 2. They have been chosen in accordance with different process dynamics when process output is increasing or decreasing. Different dynamics can be seen from series of step response shown in Fig. 3.

The identified TS model with the sampling time of $2 \mathrm{~ms}$ is

$$
\begin{aligned}
& \mathbf{R}^{1}: \quad \text { if } y(k)-y(k-10) \text { is } A_{1} \text { then } \\
& y_{\mathrm{p}}(k+1)=1.3449 y(k)+0.2288 y(k-1)-0.5822 y(k-2) \\
& +0.0188 u(k-2)+0.0199, \\
& \mathbf{R}^{2}: \text { if } y(k)-y(k-10) \text { is } A_{2} \text { then } \\
& y_{p}(k+1)=1.3174 y(k)+0.1495 y(k-1)-0.4755 y(k-2) \\
& +0.0189 u(k-2)+0.0219 \text {. }
\end{aligned}
$$

A tight fit of the simulated output (dashed) to the real response (solid) is shown in Fig. 3.

The fuzzy model from Eq. (37) was implemented in the FPFC. To design the FPFC, the reference model and the prediction horizon $H$ is needed. The third-order reference model was chosen with the respect to the model dynamics. The discrete poles of the reference model were placed on the positions:

$p_{1}=p_{2}=p_{3}=0.94$.

The prediction horizon is normally chosen to fulfil the term

$N \leqslant H \leqslant \frac{T_{\mathrm{r}}}{2 T_{\mathrm{s}}}$,

where $N$ is the process order, $T_{\mathrm{r}}$ is the time constant of reference model and $T_{\mathrm{s}}$ is the sampling time. In our case prediction horizon was chosen as $H=12$. Smaller prediction horizon on one hand results in better accuracy of the predicted process output, but on the other hand, the FPFC strongly amplifies the noise of the measured process output. 


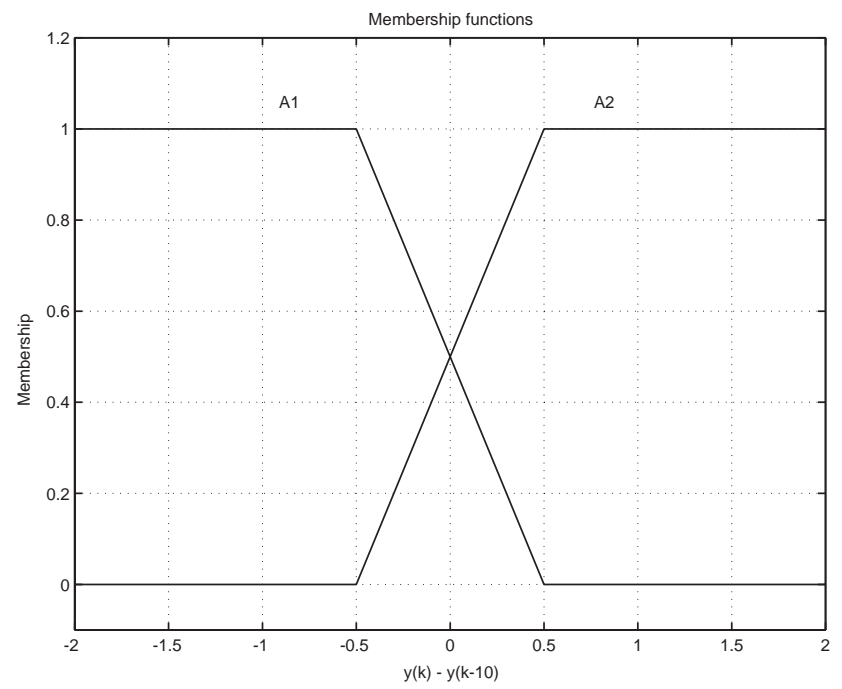

Fig. 2. Antecedent fuzzy sets.

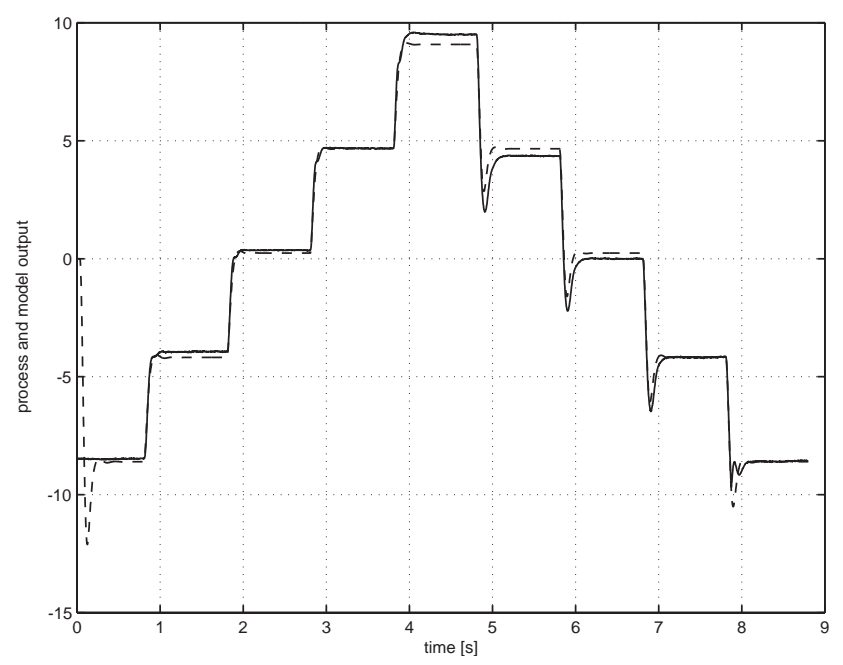

Fig. 3. Validation of the model.

Time-consuming calculating of $u(k)$ can be a problem in the case of very fast process dynamics. In each sample time the computer is dealing with different matrices $\tilde{\mathbf{A}}_{\mathrm{m}}$, $\tilde{\mathbf{B}}_{\mathrm{m}}, \quad \tilde{\mathbf{R}}_{\mathrm{m}}$ and $\tilde{\mathbf{C}}_{\mathrm{m}}$. The problem was solved in the following way: For both consequent linear models of Eq. (37) linear PFCs were designed. Matrices $\tilde{\mathbf{A}}_{\mathrm{m}}, \tilde{\mathbf{B}}_{\mathrm{m}}$, $\tilde{\mathbf{R}}_{\mathrm{m}}$ and $\tilde{\mathbf{C}}_{\mathrm{m}}$ are composed from both consequent linear models. Since the multiplication factors of all signals of control law presented in Eq. (36) are constant, can be computed in advance and represent two linear PFCs. Linear combination was made using the membership degrees $\beta_{1}$ and $\beta_{2}$. The complex matrix computations were transformed to a simple equation:

$u(k)=\beta_{1} u_{1}(k)+\beta_{2} u_{2}(k)$,

where $u_{1}(k)$ and $u_{2}(k)$ stand for output of both linear PFC.
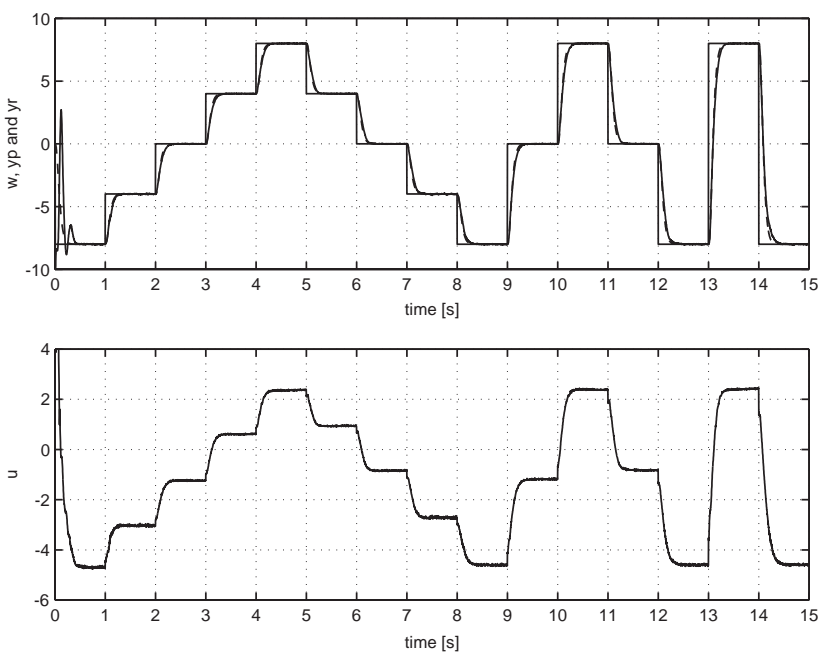

Fig. 4. Response using FPFC.
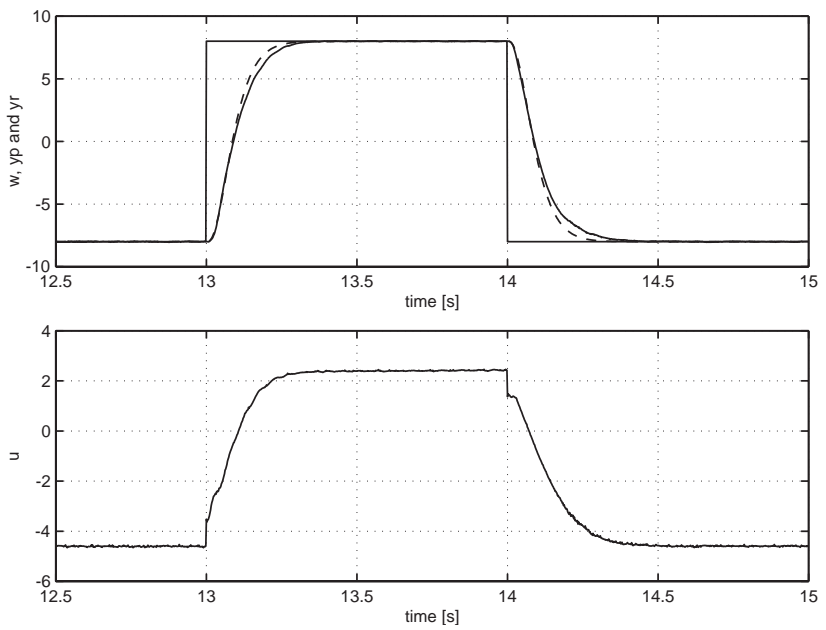

Fig. 5. Detail of Fig. 4.

In Fig. 4 the responses of the process (solid) and the reference trajectory (dashed) are shown. The detail is presented in Fig. 5.

\subsection{Comparison of FPFC with PID controller}

Finally, the comparison with a PID controller was made. The PID controller was modified. The differential input was connected directly to the process output. The structure of the PID controller can be seen from the equation as follows:

$$
\begin{aligned}
U\left(z^{-1}\right)= & K_{\mathrm{p}} E\left(z^{-1}\right)+K_{\mathrm{i}} T_{\mathrm{s}} \frac{1}{1-z^{-1}} E\left(z^{-1}\right) \\
& +\frac{K_{\mathrm{d}}}{T_{\mathrm{s}}}\left(1-z^{-1}\right) Y_{\mathrm{p}}\left(z^{-1}\right)
\end{aligned}
$$

The parameters of the PID controller were $K_{\mathrm{p}}=$ $0.185, K_{\mathrm{i}}=3.70$ and $K_{\mathrm{d}}=0.005$, and the sampling time 

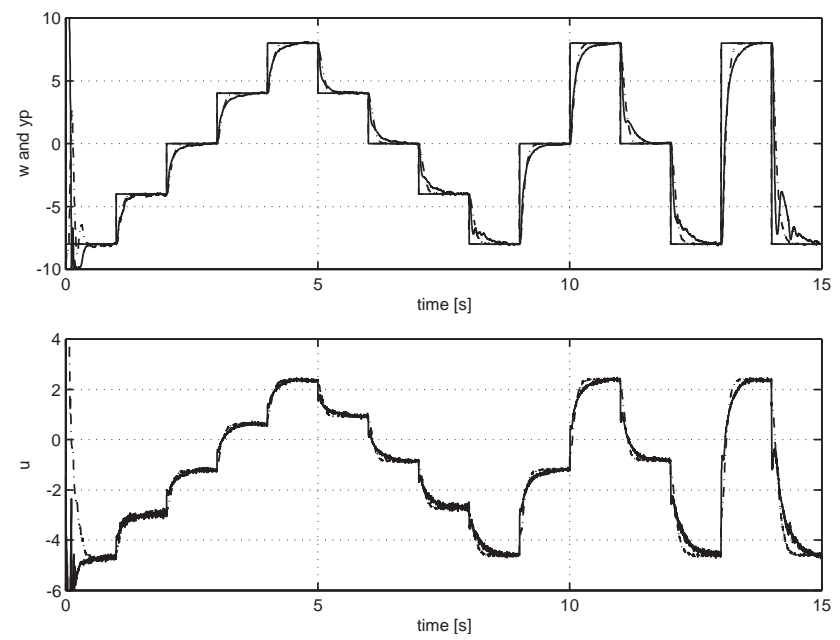

Fig. 6. Comparison with PID controller.
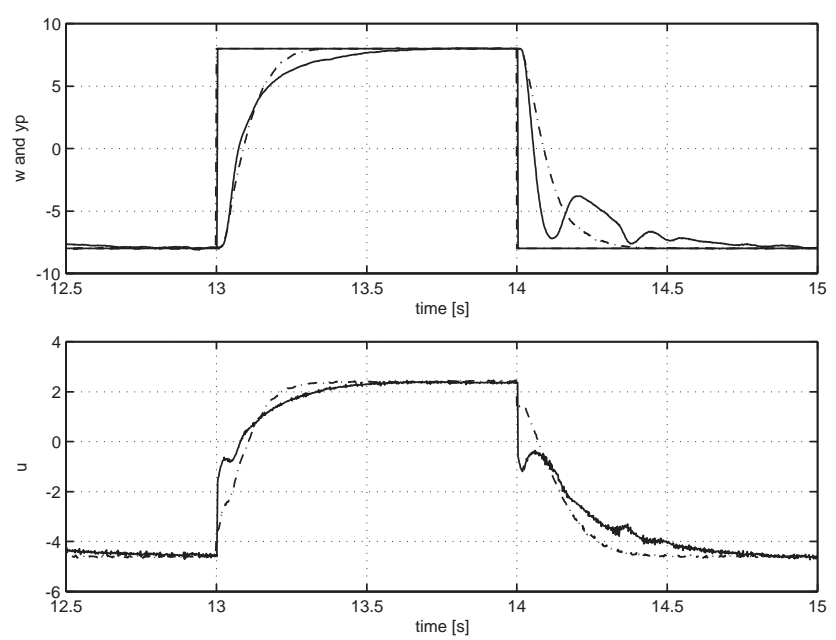

Fig. 7. Detail of Fig. 6.

was $T_{\mathrm{s}}=2 \mathrm{~ms}$. The comparison with the PID controller is shown in Fig. 6 and a detail of it in Fig. 7, where the PID response is marked with a solid line and the FPFC response with a dashed one.
In all cases the FPFC proved to be better. When using it, the settling time on positive changes of reference was twice shorter than with PID controller. Another drawback of PID controller was appearance of oscillations in the lower part of operating range.

\section{Conclusion}

The theory nonlinear prediction control law called FPFC is implemented to the nonlinear process with very fast dynamics. As seen from the comparisons with conventional PID, the prediction control together with nonlinear structure FPFC exhibits remarkable performance. Its great robustness, in the presence of model inaccuracies and unmodelled dynamics, makes FPFC convenient for a large number of applications. Regarding the idea of the magnetic bearing, the FPFC promises high-quality control.

\section{References}

Camacho, E.F., Bordons, C., 1995. Model Predictive Control in the Process Industry. Springer, London.

Clarke, D.W., Mohtadi, C., Tuffs, P.S., 1987. Generalized Predictive Control-Part 1, Part 2. Automatica 24, 137-169.

Cutler, C.R., Ramaker, B.L., 1980. Dynamic matrix control-a computer control algorithm. ACC, San Francisco.

Richalet, J., Rault, A., Testud, J.L., Papon, J., 1978. Model predictive heuristic control: applications to industrial processes. Automatica $14,413-428$.

Škrjanc, I., Matko, D., 2000. Predictive functional Control based on fuzzy model for heat-exchanger pilot plant. IEEE Transactions on Fuzzy Systems 8 (6), 705-712.

Škrjanc, I., Matko, D., 2001. Fuzzy predictive functional control in the state space domain. Journal of Intelligent and Robotic Systems 31 (1), 283-297.

Sugeno, M., Tanaka, K., 1991. Successive Identification of a fuzzy model and its application to prediction of a complex system. Fuzzy Sets and Systems 42, 315-334.

Takagi, Sugeno, 1985. Fuzzy identification of systems and its applications to Modelling and Control: IEEE transactions on Systems, Man, and Cybernetics (15), 116-132. 